PROCEEDINGS OF THE

AMERICAN MATHEMATICAL SOCIETY

Volume 134, Number 12, December 2006, Pages 3605-3611

S 0002-9939(06)08442-5

Article electronically published on June 9, 2006

\title{
FIXED POINTS OF UNIVALENT FUNCTIONS II
}

\author{
GERALD SCHMIEDER \\ (Communicated by Juha M. Heinonen) \\ To my friend Masakazu Shiba on the occasion of his 60th birthday
}

\begin{abstract}
For a closed nowhere dense subset $C$ of $\partial \mathbb{D}$ a bounded univalent holomorphic function $f$ on $\mathbb{D}$ is found such that $C$ equals the cluster set of its fixed points.
\end{abstract}

\section{INTRODUCTION}

Let $f$ be a univalent (i.e. holomorphic and injective, but not necessarily normalized) function of the open unit disk $\mathbb{D}$ in the complex plane $\mathbb{C}$.

Such a function $f \not \equiv i d_{\mathbb{D}}$ may have infinitely many fixed points. In 2 it is proved that for each Blaschke sequence $\left(z_{n}\right)$ there exists a univalent function $f \not \equiv i d_{\mathbb{D}}$ with $f\left(z_{n}\right)=z_{n}$ for all $n \in \mathbb{N}$, provided that the cluster set $F_{c}(f)$ of $\left(z_{n}\right)$ is an arbitrary Carleson set, i.e. a closed set of Lebesgue measure 0 on $\partial \mathbb{D}$ with $\sum_{n} \varepsilon_{n} \log \varepsilon_{n}>-\infty$, where $\varepsilon_{n}$ denotes the length of the open components (arcs) of its complement with respect to $\partial \mathbb{D}$. In 4 it is proved that $F_{c}$ is a nowhere dense subset of the unit circle unless $f \equiv i d_{\mathbb{D}}$. It remains open whether $F_{c}(f)$ can be a proper subset of $\partial \mathbb{D}$ of positive measure. Here we will prove:

Theorem 1. Let $C$ be a nowhere dense, closed subset of $\partial \mathbb{D}$. Then there exists a bounded univalent function $g: \mathbb{D} \rightarrow \mathbb{C}$ with the property that the cluster set $F_{c}(g) \subset \partial \mathbb{D}$ of its fixed points is $C$.

Cantor sets on $\partial \mathbb{D}$ fulfill the assumption of Theorem[1] Their Lebesgue measure with respect to $\partial \mathbb{D}$ can be arbitrarily close to $2 \pi$.

If $f: \mathbb{D} \rightarrow \mathbb{C}$ is holomorphic and $\alpha \in\left[0,2 \pi\left[\right.\right.$, then the cluster set $C_{\alpha}(f)$ of the restriction $f_{\alpha}(t):=f\left(t e^{i \alpha}\right)$ for $t \rightarrow 1-$ is called the radial cluster set of $f$ with respect to $e^{i \alpha}$. It is well known that this cluster set coincides with the cluster set of $f(z)$, where $z$ tends to $e^{i \alpha}$ in an arbitrary triangle $\Delta_{\alpha} \subset \mathbb{D}$ with $e^{i \alpha} \in \partial \Delta_{\alpha}$. One can also describe $C_{\alpha}(f)$ as the set of principal points of the prime end of the image domain $f(\mathbb{D})$ which belongs to the boundary point $e^{i \alpha}$ of $\mathbb{D}$ (see [5] or [6]). An accumulation point of $f(z)$ for $z \rightarrow e^{i \alpha}$ which is not radial is called tangential. In terms of prime ends of $f(\mathbb{D})$ these are the points of the corresponding prime end impressions which are not principal points.

Received by the editors December 14, 2004 and, in revised form, June 30, 2005.

2000 Mathematics Subject Classification. Primary 30C35, 30D40.

Key words and phrases. Univalent functions, fixed points, cluster set.

(C)2006 American Mathematical Society

Reverts to public domain 28 years from publication 
By a result in 4] (Thm. 1), which combines a result of Beurling (1], Thm. 3.5) and the theorem of F. and M. Riesz ([1, Thm. 2.5), the set $\left\{e^{i \alpha}: e^{i \alpha} \in C_{\alpha}(f)\right\}$ has measure zero if $f: \mathbb{D} \rightarrow \mathbb{C}$ is univalent. This shows that the fixed points of the function $f$ in Theorem 1 have to approach their accumulation points on the boundary of $\mathbb{D}$ tangentially up to a zero set.

The crucial tool in the proof is Theorem 2, which states the possibility of fusion of univalent functions. This is a consequence of an improved version of Alice Roth's well-known fusion lemma, due to Masakazu Shiba and the author (7], 8]).

\section{FUSiON OF UNIVALENT FUNCTIONS}

In this section we will prove the following result.

Theorem 2. Let $G_{1}, G_{2}$ be simply connected domains, bounded by Jordan curves $\gamma_{1}$, resp. $\gamma_{2}$, and $\overline{G_{1}} \cap \overline{G_{2}}=\left\{z_{0}\right\}$. Moreover let $f_{1}$, resp. $f_{2}$, be continuous on $\overline{G_{1}}$, resp. on $\overline{G_{2}}$, univalent on $G_{1}$, resp. on $G_{2}$.

If $f_{1}\left(\overline{G_{1}}\right) \cap f_{2}\left(\overline{G_{2}}\right)$ consists of the single point $w_{0}=f_{1}\left(z_{0}\right)=f_{2}\left(z_{0}\right)$, then, for all $\varepsilon>0$, there exists a neighborhood $U$ of $\overline{G_{1}} \cup \overline{G_{2}}$ and an entire function $f$, which is univalent on $U$ and fulfills $\left\|f-f_{1}\right\|_{G_{1}}<\varepsilon$ as well as $\left\|f-f_{2}\right\|_{G_{2}}<\varepsilon$.

The proof requires some preparation.

Definition 1. We call a pair $K_{1}, K_{2}$ of compact sets in the plane normal, if

(1) $\mathbb{C} \backslash\left(K_{1} \cup K_{2}\right)$ is connected.

(2) The interiors $K_{1}^{o}, K_{2}^{o}$ of both sets are connected.

(3) $K_{1} \cap K_{2}^{o}=K_{2} \cap K_{1}^{o}=\emptyset$.

(4) $K_{1}=\overline{K_{1}^{o}}$ and $K_{1}=\overline{K_{1}^{o}}$.

Definition 2. We say that a pair of compact plane sets $K_{1}, K_{2}$ has the rational fusion property if there exists some positive number $a=a\left(K_{1}, K_{2}\right)$ such that the following is true: For each pair $r_{1}, r_{2}$ of rational functions and for each compact set $K$ there is some rational function $r$ with

$$
\left|r(z)-r_{j}(z)\right| \leq a \cdot \sup \left\{\left|r_{1}(w)-r_{2}(w)\right|: w \in K \cup\left(K_{1} \cap K_{2}\right)\right\}
$$

for all $z \in K_{j} \cup K$ simultaneously for $j=1,2$.

We say that the pair $K_{1}, K_{2}$ has the holomorphic fusion property with respect to some domain $G \subset \mathbb{C}$ if we may replace the rational functions $r_{1}, r_{2}, r$ by holomorphic functions on $G$.

In [7] the following extension of Alice Roth's famous Fusion Lemma (cf. [3]) is proved, basing on a result of M. Shiba and the author 8 .

Proposition 1. A normal pair of compact sets $K_{1}, K_{2}$ has the rational fusion property if and only if $\partial K_{1} \cup \partial K_{2}=\partial\left(K_{1} \cup K_{2}\right)$.

Proof of Theorem 2. Let $\gamma_{1, \delta}$ be a Jordan curve in $\mathbb{C} \backslash \overline{G_{1}}$ with dist $\left(\gamma_{1}, \gamma_{1, \delta}\right)<\delta$ and let $G_{1, \delta}$ denote the bounded component of $\mathbb{C} \backslash \gamma_{1, \delta}$. Then $\overline{G_{1}} \subset G_{1, \delta}$, and we have a biholomorphic map $\phi_{\delta}: G_{1, \delta} \rightarrow G$. An equicontinuity argument shows that, for each given $\varepsilon$, the function $f_{1, \delta}=f_{1} \circ \phi_{\delta}$ fulfills $\left\|f_{1}-f_{1, \delta}\right\|_{G_{1}}<\varepsilon$ if $\delta \leq \delta_{0}(\varepsilon)$. We may also assume that $f_{1, \delta}\left(z_{0}\right)=f_{1}\left(z_{0}\right)$, otherwise we replace $\varepsilon$ by $\varepsilon / 2$ and consider 
the function $f_{1, \delta}\left(z_{0}\right)+f_{1}\left(z_{0}\right)-f_{1, \delta}\left(z_{0}\right)$. This shows that we may provide that $f_{1}$ (as well as $f_{2}$ ) is univalent on a neighborhood of $K_{1}:=\overline{G_{1}}$ (resp. on $K_{2}=\overline{G_{2}}$ ).

Without loss of generality we may assume that $z_{0}=0, f_{1}(0)=0=f_{2}(0)$.

In the first part of the proof we consider the case that $f_{1}{ }^{\prime}(0)=f_{2}{ }^{\prime}(0)$. Without loss of generality we may assume that $f_{1}, f_{2}$ are entire functions, because we may approximate our starting functions uniformly on suitable compact sets containing $K_{1}$, resp. $K_{2}$, in its interior. This will preserve the univalence on a neighborhood of $K_{j}$ (as the argument principle shows) if the approximation bound is sufficiently small.

Obviously $K_{1}, K_{2}$ is a normal pair, and we also find a normal pair of compact sets $C_{j}$, also bounded by a Jordan curve respectively, containing $K_{j} \backslash\{0\}(j=1,2)$ in the interior, such that $C_{1} \cap C_{2}=\{0\}$, and $f_{1}, f_{2}$ are univalent in an open neighborhood of $C_{1}, C_{2}$. We may also provide that $C_{1}, C_{2}$ is a rational fusion pair by Proposition 1 and, obviously, that it is a holomorphic fusion pair. As the "bridge" $K$ joining $C_{1}, C_{2}$ we take a disc $D_{\rho}:=\{|z| \leq \rho\}$; its radius will be fixed later. So we find some entire function $f$ with the property that

$$
\left|f^{\prime}(z)-f_{j}^{\prime}(z)\right| \leq a \cdot \max \left\{\left|f_{1}^{\prime}(w)-f_{2}{ }^{\prime}(w)\right|: w \in D_{\rho}\right\} \quad\left(z \in C_{j} \cup D_{\rho}, j=1,2\right),
$$

where $a$ depends only on $C_{1}, C_{2}$. This shows that (note that $f_{1}(0)=f_{2}(0)$ has been assumed)

$$
\left|f(z)-f_{j}(z)\right| \leq b \cdot \max \left\{\left|f_{1}^{\prime}(w)-f_{2}{ }^{\prime}(w)\right|: w \in D_{\rho}\right\} \quad\left(z \in C_{j} \cup D_{\rho}, j=1,2\right)
$$

with some $b$ depending only on geometrical properties of $C_{1}, C_{2}$. Thus, by the argument principle, we see that $f$ is univalent on a neighborhood of $C_{1}$ as well as on a neighborhood of $C_{2}$ if $\max \left\{\left|f_{1}{ }^{\prime}(w)-f_{2}{ }^{\prime}(w)\right|: w \in D_{\rho}\right\}$ is sufficiently small, and thus for small $\rho$, as follows from the assumption that $f_{1}{ }^{\prime}(0)=f_{2}{ }^{\prime}(0)$ and from the continuity of $f_{j}{ }^{\prime}$.

On $D_{\rho}$ we have for small $\rho$ that $\left|f^{\prime}(z)-f_{1}{ }^{\prime}(0)\right|<\left|f_{1}{ }^{\prime}(0)\right|$. Since $f_{1}{ }^{\prime}(0) \neq 0$ this implies that $\Re f^{\prime}>0$ on $D_{\rho}$, and a well-known argument shows that therefore $f$ is univalent on $D_{\rho}$. Finally we may assume that $f\left(C_{1} \backslash D_{\rho}\right) \cap f\left(C_{2} \backslash D_{\rho}\right)=\emptyset$, as follows from the assumption that $f_{1}\left(K_{1}\right) \cap f_{2}\left(K_{2}\right)=\{0\}$, provided that the approximation was done with a bound sufficiently close to zero.

So $f$ has the desired properties if $\rho$ is small enough.

Now we consider the case that $f_{1}^{\prime}(0) \neq f_{2}{ }^{\prime}(0)$.

Let $\sigma(z)=\sigma(z, \eta)=1-(1-z)^{\eta}$ with some (small) $\eta>0$. This function is holomorphic on each simply connected domain which does not contain 1 . We obtain $\sigma^{\prime}\left(1-\eta^{1 /(1-\eta)}, \eta\right)=1$. Without loss of generality we may assume that $1 \notin K_{1}$.

Now we consider the functions $\varphi(z, \eta)=\sigma\left(z+1-\eta^{1 /(1-\eta)}, \eta\right)$ and $g_{1}(z)=$ $f_{1}(z)+\left(f_{2}^{\prime}(0)-f_{1}^{\prime}(0)\right) \varphi(z, \eta)$ on $K_{1}$ for small $\eta$. Note that this expression is defined on a neighborhood of $K_{1}$. Then, by the mentioned properties of $\sigma$, we have that

$$
\|\varphi(., \eta)\|_{K_{1}}=|\varphi(0)|=1-\eta^{\eta /(1-\eta)} \rightarrow 0 \text { if } \eta \rightarrow 0
$$

provided that $\eta=\eta(K, \varepsilon)$ is small. Then $g_{1}$ is univalent on a neighborhood of $K_{1}$ for all small $\eta$, and if some $\varepsilon$ is given, we have that $\left\|g_{1}-f_{1}\right\|_{K_{1}}<\varepsilon$ for those $\eta$. On the other hand we see that $g_{1}{ }^{\prime}(0)=f_{2}{ }^{\prime}(0)$. The remaining argumentation is as in the first case, if we replace $f_{1}$ by $g_{1}$. 


\section{A PREPARATORY LEMMA ON CONFORMAL MAPPINGS}

We will need the following statement on conformal mappings.

Lemma 1. Let $D=D_{\kappa}=\{|z-1|<\kappa\}(\kappa>0)$ and let $H$ be a (simply connected) Jordan domain with $H \subset \mathbb{D} \cup D$ and $\mathbb{D} \backslash D \subset H$. The set $H \cap \partial \mathbb{D} \subset D$ is assumed to be nonempty and connected.

Let $\Theta$ denote the Riemann map of $\mathbb{D}$ onto $H$ with $\Theta(0)=0$ and let some $\varepsilon>0$ be given. With respect to the continuous extension of $\Theta$ on $\overline{\mathbb{D}}$ we define the set

$$
E=\{z:|z|=1 \text { and }|\Theta(z)|>1\} .
$$

Then $E$ is an interval on $\partial \mathbb{D}$, and there exists some $\kappa_{0}>0$ such that the length of $E$ is less than $\varepsilon$ if $\kappa<\kappa_{0}$.

Proof. That $E$ is an interval on $\partial \mathbb{D}$ follows from the assumption on $H \cap \partial \mathbb{D} \subset D$.

If the assertion were not true, we could find some positive $\varepsilon$ and a sequence $\delta_{k} \rightarrow 0, \kappa_{k} \rightarrow 0$ and simply connected domains $H_{k}$ with the described properties, such that the lengths of the intervals $E_{k}$ (defined with respect to the associated conformal maps $\Theta_{k}$ with $\left.\Theta_{k}(0)=0\right)$ all are at least $\varepsilon$. Let $V$ be a disc centered at 1. Then it follows by standard arguments that the sequence of the conformal maps $\Theta_{k}$ converges uniformly on $\overline{\mathbb{D}} \backslash V$ to the identity map. Thus $E_{k}$ must be contained in $V$ for all sufficiently large $k$. This leads to a contradiction if we take $V$ so small that the length of $\partial \mathbb{D} \cap V$ is less than $\varepsilon$.

\section{A sequence of functions}

Let $C \subset \partial \mathbb{D}$ be a nowhere dense closed set. Then the complement with respect to $\partial \mathbb{D}$ is open, and thus is the countable union of pairwise disjoint open intervals $I_{j}$ on $\partial \mathbb{D}$. Let $e^{i \alpha_{j}}$ be the center point of $I_{j}$. For each $j \in \mathbb{N}$ we fix some open disk $V_{j}$ with $e^{i \alpha_{j}} \in V_{j}$ and $V_{j} \cap \partial \mathbb{D} \subset I_{j}$. Moreover we may assume that these $V_{j}$ are pairwise disjoint.

Remark 1. If a sequence $w_{j} \in \overline{V_{j}} \cap \mathbb{D}(j \in \mathbb{N})$ is given, then the cluster set of $\left(w_{j}\right)$ consists of all nonisolated points of $C$, as follows from the properties of $C$ as a closed and nowhere dense set. In order to construct a function $g$ which also fulfills the assertion of Theorem 10 with respect to possible isolated points of $C$, we can enlarge this set by adding a discrete subset of $\partial \mathbb{D}$, which contains for each isolated point $c_{1} \in C$ a sequence $c_{1}^{\nu} \in \partial \mathbb{D} \backslash C$ tending to $c_{1}$. Then the fixed point cluster $F_{c}(g)$ of the function $g$ we will construct below contains $c_{1}$, but not the additional points $c_{1}^{\nu}$. We may assume in the following that $C$ has been prepared in this way.

Now we describe a sequence of positive numbers $\varepsilon_{j}(j \geq 2)$ with $\varepsilon_{2}>\varepsilon_{3}>\ldots$ and $\sum_{j=1}^{\infty} \varepsilon_{j}<\frac{1}{3}$.

The aim of this section is to construct a sequence of functions $g_{n}: \mathbb{D} \rightarrow \mathbb{C}$ which fulfills:

(i) $g_{n}$ is holomorphic and univalent on $\overline{\mathbb{D}}$.

(ii) $\mid g_{n}\left(\underline{z)-g_{n-1}}(z) \mid<\varepsilon_{n}\right.$ for all $z \in \mathbb{D} \backslash V_{n}$ if $n>1$.

(iii) $\mathbb{C} \backslash \overline{g_{n}(\mathbb{D})}$ is connected.

(iv) $e^{i \alpha_{j}} \notin g_{n}(\mathbb{D})$ for $j>n$.

(v) For each $n \in \mathbb{N}$ the function $g_{n}$ has fixed points $z_{n, k} \in V_{k}(k=1, \ldots, n)$, and it holds that $\left|z_{n, k}-z_{k, k}\right|<\frac{1}{2} \operatorname{dist}\left(z_{k, k}, \partial V_{k}\right)$ for all $k=1, \ldots, n-1$. 
(vi) $g_{n}$ has in $\overline{\mathbb{D}} \backslash V_{n}$ the same number of fixed points as $g_{n-1}$ for all $n \geq 2$.

(vii) $g_{n}$ has no fixed point on $\partial \mathbb{D}(n \in \mathbb{N})$.

We try $g_{1}(z):=\frac{z}{4}+e^{i \alpha_{1}} \cdot \sigma\left(\frac{z e^{-i \alpha_{1}}}{1+\eta}, \eta\right)$ with $0<\eta<1$ (the functions $\sigma(., \eta)$ have been defined close to the end of section 2). The derivative of this function has, for each choice of the parameters, positive real part in $\mathbb{D}$, and therefore it is univalent. The number $\eta$ can be taken in such a way that $g_{1}$ has a fixed point $z_{1,1}=\rho_{1} e^{i \alpha_{1}}$ in $V_{1}$. Concerning (v) we having nothing more to check in this first step. Note that $\sigma(. \eta)$ tends uniformly to 0 on each compact subset of $\mathbb{C} \backslash[1, \infty[$. The conditions (i), (iii), (iv) and (vii) are obviously fulfilled for all sufficiently small positive $\eta$, while (ii) and (vi) are empty in this case. If some number $b$ bigger than $\frac{1}{4}$ is given we can arrange that $\left|g_{1}\right|<b$ on $\mathbb{D} \backslash V_{1}$.

Let, for some $n \in \mathbb{N}$, a function $g_{n}$ with the mentioned properties be found, and we will construct the function $g_{n+1}$.

We start with this part of $(\mathrm{v})$, which claims that there are appropriate fixed points $z_{n+1,1}, \ldots, z_{n+1, n}$ of our candidate function $F$. The "new" fixed point $z_{n+1, n+1}$ we will discuss later. From (v) we know that $g_{n}$ has a fixed point $z_{n, k}$ in each of the the discs

$$
\Delta_{k}:=\left\{\left|z-z_{k, k}\right|<\frac{1}{2} \operatorname{dist}\left(z_{k, k}, \partial V_{k}\right)\right\} \quad(k=1, \ldots, n) .
$$

Since a fixed point of a function $f$ is a solution of the equation $f(z)-z=0$, we know that 0 is an inner point of every domain $W_{k}:=\left(g_{n}-i d\right)\left(\Delta_{k}\right)(k=1, \ldots, n)$. So, if $\varepsilon<\operatorname{dist}\left(z_{n, k}, \partial W_{k}\right)$ for all $k=1, \ldots, n$, we indeed find fixed points $z_{n+1, k} \in$ $\Delta_{k} \subset V_{k}(k=1, \ldots, n)$ of $F$, provided that $\left|F-g_{n}\right|<\varepsilon$ on $\Delta_{1} \cup \cdots \cup \Delta_{n}$. From (vii) we conclude that the fixed point set of $g_{n}$ in $\overline{\mathbb{D}}$ has positive distance $d$ to $\partial \mathbb{D}$. Therefore we will approximate $g_{n}$ with a bound

$$
\varepsilon<\min \left\{\varepsilon_{n+1}, \min _{k=1, \ldots, n} \operatorname{dist}\left(z_{n, k}, \partial W_{k}\right), d\right\} .
$$

Now we fix some $\varepsilon$ according to this condition. Note that each holomorphic $F$ : $\overline{\mathbb{D}} \rightarrow \mathbb{C}$ with $\left\|F-g_{n}\right\|_{\mathbb{D} \backslash V_{n+1}}<\varepsilon$ will have fixed points $z_{n+1,1}, \ldots, z_{n+1, n}$ with the properties mentioned in (v). Moreover, the number of fixed points in $\overline{\mathbb{D}} \backslash V_{n+1}$ of such a function $F$ is the same as of $g_{n}$ in this set. Thus (vi) is automatically fulfilled if we take $F$ as described.

In particular, $F$ cannot have any fixed point in $\partial \mathbb{D} \backslash V_{n+1}$, as follows from (vii).

Now we consider the point $z_{0}:=e^{i \alpha_{n+1}}$. Then $z_{0} \in \mathbb{C} \backslash \overline{g_{n}(\mathbb{D})}$ by (v). Since $z_{0} \notin V_{1} \cup \cdots \cup V_{n}$ it follows from (ii) that $\left|g_{n}\left(z_{0}\right)\right| \leq\left|g_{1}\left(z_{0}\right)\right|+\sum_{j=2}^{n} \varepsilon_{j} \leq \frac{1}{3}+\frac{1}{3}$.

By (iii) we find some smooth Jordan arc

$$
\Gamma:[0,1] \rightarrow \mathbb{C} \backslash \bigcup_{j \in \mathbb{N} \backslash\{n+1\}} \overline{V_{j}}
$$

with $\Gamma(0)=g_{n}\left(z_{0}\right), \Gamma(1)=z_{0}$ and $\Gamma(t) \in \mathbb{C} \backslash \overline{g_{n}(\mathbb{D})}$ for all $\left.\left.t \in\right] 0,1\right]$.

Moreover there exists some simply connected domain

$$
V \subset \mathbb{C} \backslash\left(\overline{g_{n}(\mathbb{D})} \cup \underset{j \in \mathbb{N} \backslash\{n+1\}}{\bigcup_{j}} \bar{x}\right.
$$

(think of $V$ as a small tube containing $\Gamma(] 0,1])$ ) with $\partial V \cap \partial g_{n}(\mathbb{D})=\left\{g_{n}\left(z_{0}\right)\right\}$.

Remark 2. The domain $V$ depends only on $g_{n}$ and on $z_{0}$, and it contains some closed disc $D^{*}=\left\{\left|z-z_{0}\right| \leq r\right\}$ of positive radius. 
We find, by Riemann's mapping theorem, some surjective univalent function

$$
f_{1}: G_{1}:=\left\{z:\left|z-\left(1+\frac{\kappa}{4}\right) z_{0}\right|<\frac{\kappa}{4}\right\} \rightarrow V
$$

with $f_{1}\left(z_{0}\right)=g_{n}\left(z_{0}\right)$, where the parameter $\kappa>0$ is arbitrary in the moment, but it will be fixed below. With the notations $K_{1}=\overline{G_{1}}, G_{2}=\mathbb{D}, K_{2}=\overline{G_{2}}$ and $f_{2}=g_{n}$ we obtain:

(a) $\Gamma([0,1]) \subset f_{1}\left(K_{1}\right)$,

(b) $G_{1}$ and $G_{2}$ are Jordan domains,

(c) $K_{1} \cap K_{2}=\left\{z_{0}\right\}$,

(d) $f_{1}$, resp. $f_{2}$, is continuous on $K_{1}$, resp. on $K_{2}$, and univalent on $G_{1}$, resp. on $G_{2}$,

(e) $f_{1}\left(K_{1}\right) \cap f_{2}\left(K_{2}\right)=\left\{g_{n}\left(z_{0}\right)\right\}$ and $g_{n}\left(z_{0}\right)=f_{1}\left(z_{0}\right)=f_{2}\left(z_{0}\right)$.

We apply Theorem 2 and find an entire function $f$, which is univalent on a neighborhood $U$ of $K_{1} \cup K_{2}$ and fulfills

$$
\left\|f_{1}-f\right\|_{K_{1}}<\frac{\varepsilon}{2} \text { and }\left\|f_{2}-f\right\|_{K_{2}}<\frac{\varepsilon}{2} .
$$

Let $H \subset \mathbb{D} \cup\left\{\left|z-z_{0}\right|<\kappa\right\}$ be a simply connected Jordan domain such that $\mathbb{C} \backslash \bar{H}$ is connected and that $f$ is univalent on $H$.

Now we apply Lemma 1 and consider the conformal map $\Theta: \mathbb{D} \rightarrow H$. We will have that

$$
\|\Theta-i d\|_{\mathbb{D}}<\frac{\varepsilon}{2} \text { and } G_{1} \subset \Theta\left(V_{n+1} \cap \mathbb{D}\right),
$$

if $\kappa$ is small enough, and for small $\kappa$ we also see that $F^{-1}\left(D^{*}\right) \subset D^{*}$, where $F:=f \circ \Theta$ (which is our candidate for the desired function $g_{n+1}$ ). We fix such a number $\kappa$.

$F$ is continuous on $\overline{\mathbb{D}}$ and univalent on $\mathbb{D}$ by construction. This gives (i).

On $\mathbb{D} \backslash V_{n+1}$ we see that $\left|F(z)-g_{n}(z)\right|<\varepsilon \leq \varepsilon_{n+1}$ by (2) and (3). So (ii) is fulfilled (with $F$ as the candidate for $g_{n+1}$ ).

$\mathbb{C} \backslash \overline{F(\mathbb{D})}$ is the complement of the closure of the domain $H$, and thus this set is connected by construction. This shows that (iii) holds.

Since $e^{i \alpha_{j}} \in V_{j}(j \in \mathbb{N})$ we verify (iv) by (11).

Now we prove the part of (v) concerning the desired fixed point $z_{n+1, n+1}$ of $F$ in $V_{n+1}$.

It has been remarked that the closed disc $D^{*}$ is mapped under $F^{-1}$ into itself. By Brouwer's fixed point theorem, $F^{-1}$ has a fixed point $z_{1}$ in $D^{*}$. But then $z_{1}=F^{-1}\left(z_{1}\right) \in V_{n+1}$ by (3) and by $D^{*} \subset V$.

Finally we discuss property (vii) with respect to $F$. We discuss the $h(z):=F(z)-$ $z$. For each $\delta>0$ there is of course some complex number $w_{0}$ with $w_{0} \notin h(\partial \mathbb{D})$. If we consider, for suitably small $\delta$, the function $F(z)-w_{0}$ instead of $F$, then (vii) is fulfilled and (i),...,(vi) remain true.

Now we are ready to define $g_{n+1}:=F$ and $z_{n+1, n+1}:=z_{1}$.

The construction of the functions $g_{n}$ can be done in such a way that, for example, $\left|g_{n}(z)\right|<2$ for all $n \in \mathbb{N}$ and for all $z \in \mathbb{D}$. The bound 2 could be replaced by each number bigger than 1 . 


\section{Proof of Theorem 1}

From (ii) and $\sum_{j=1}^{\infty} \varepsilon_{j}<\frac{1}{3}$ we see that $g=\lim _{n \rightarrow \infty} g_{n}$ exists, and $g$ can be neither the identity nor a constant function. By standard arguments we conclude that $g$ is univalent on $\mathbb{D}$, and it follows from the construction that $|g|$ is bounded. The inequality

$$
\left|z_{n, k}-z_{k, k}\right|<\frac{1}{2} \operatorname{dist}\left(z_{k, k}, \partial V_{k}\right)
$$

for all $1 \leq k<n$ in (v) guarantees that none of the sequences $s_{k}=\left(z_{n, k}\right)_{n \geq k}$ can tend to $\partial \mathbb{D}$. Thus $g$ has in each $V_{k}$ a fixed point $z_{k}$, which obviously fulfills $\left|z_{k}-z_{k, k}\right| \leq \frac{1}{2} \operatorname{dist}\left(z_{k, k}, \partial V_{k}\right)$.

The fixed point cluster $F_{c}(g)$ of the function $g$ contains the cluster set of the sets $V_{k}$, which is $C$ (or, to be more precise, the set of all nonisolated points of $C$, compare Remark 1).

The validity of the opposite inclusion can be obtained as follows. Let some $\zeta \in \partial \mathbb{D}$ be given, which is not a nonisolated point of $C$ (compare Remark 1). Then there is some open disk $U(\zeta)$, centered at $\zeta$, and some $n_{0} \in \mathbb{N}$ with $U(\zeta) \cap V_{n}=\emptyset$ for all $n \geq n_{0}$. Therefore, by (vi), we see that the number of fixed points of $g_{n}$ in $U(\zeta) \cap \mathbb{D}$ has a (with respect to $n$ ) uniform finite bound. Thus $\zeta$ does not belong to $F_{c}(g)$.

\section{ACKNOWLEDGEMENT}

I thank the referee for valuable suggestions and comments.

\section{REFERENCES}

[1] Collingwood, E.F., Lohwater, A.J.: The theory of cluster sets. Cambridge University Press, Cambridge 1966. MR0231999 (38:325)

[2] Doppel, K., Köditz, H., Timmann, S.: Bemerkungen über Fixpunktmengen schlichter Funktionen. Rend. Ist. di Matem. Univ. di Trieste vol. VII, fasc. II (1976), 161-165. MR0463424 $(57: 3375)$

[3] Gaier, D.: Lectures on complex approximation (translated from the german). Birkhäuser, Boston-Basel-Stuttgart 1987. MR0894920 (88i:30059b)

[4] Gharibyan, T., Schmieder, G.: Fixed Points of Univalent Funktions. Computational Methods and Function Theory 3 (2003), 299-304. MR.2082019 (2005d:30049)

[5] Ohtsuka, M.: Dirichlet problem, extremal length and prime ends. Van Nostrand, LondonToronto-Melbourne 1970.

[6] Pommerenke, C.: Univalent functions. Vandenhoeck \& Ruprecht, Göttingen 1975. MR $0507768(58: 22526)$

[7] Schmieder, G.: Fusion lemma and boundary structure. J. Approx. Theory 71 (1992), 305-311. MR.1191577 (94a:30032)

[8] Schmieder, G., Shiba, M.: Über ein Lemma der komplexen Approximationstheorie. Manuscripta Math. 65 (1989), 447-464. MR.1019702 (90j:30064)

Fakultät V, Institut für Mathematik, Universität Oldenburg, D-26111 Oldenburg, Germany 